\title{
Un ejemplo de oxímoron en música: el indie en España, una escena comercial*
}

\author{
An Example of an Oxymoron in Music: \\ Indie in Spain, a Commercial Scene
}

Desde su aparición, la etiqueta indie o independiente se ha convertido en una de las categorías más dificiles de definir dentro de las músicas populares urbanas en España, sobre todo porque en sentido estricto no debería considerarse un género o un estilo musical. Este artículo pretende abordar el estudio de este controvertido término, definirlo, enfrentarlo a los conceptos de género musical y escena, y analizar toda la problemática que rodea a esta etiqueta cambiante, prestando especial atención a muchas de sus contradicciones, siendo la más relevante su particular vinculación con la industria discográfica.

Palabras clave: música popular urbana, indie, independiente, escena comercial.

Since its appearance, the term indie or independent has become one of the most difficult categories to define in urban popular music in Spain, especially because it shouldn't strictly be considered an independent musical genre or style. This article examines this controversial term, defining it, confronting it with the concepts of musical genre and scene, and analysing the whole problem surrounding this varying term. Particular attention will be paid to many of its contradictions, the most important being its particular association with the recording industry.

Keywords: urban popular music, indie, independent, commercial scene.

En 1992, Julio Ruiz, periodista y locutor de Radio Nacional de España 3, utilizó el término indie, abreviación de independent o independiente, para referirse a un grupo de bandas y artistas españoles sin contrato discográfico que participaban en un concurso de maquetas que se emitía en su programa Discogrande ${ }^{1}$. En esta competición, el público tuvo un papel activo y llegó a identificarse con las formaciones que participaron en el evento. Esto suscitó el interés de la industria discográfica y algunas de las bandas concursantes firmaron contratos con compañías multinacionales como la Radio Corporation of America (RCA), caso de Los Planetas².

Esto condujo a una paradoja: a pesar de que ya no eran independientes en sentido comercial estas bandas siguieron siendo etiquetadas de manera

\footnotetext{
* Este trabajo se enmarca dentro del proyecto I+D Microhistoria de la música española contemporánea (HAR2015-69931-C3-1-P I).

${ }^{1}$ José Antonio Rueda: Independientes [Grabación audiovisual], Almería, La Fabriquilla de Producción Audiovisual, 2012.

${ }^{2}$ Fernando Barrera: Hibridación, globalización y tecnología: flamenco y música indie en Andalucía (19772012), tesis doctoral, Granada, Universidad de Granada, 2014, p. 127.
} 
recurrente como indies por prensa y público, diversificando la utilización original que se había dado al término de origen anglosajón en nuestro país.

Precisamente, el denominador indie a nivel internacional ya poseía una importante trayectoria cuando comenzó a usarse en España, y a lo largo de su existencia había reflejado esta diversificación. Durante la segunda mitad del siglo XX había sido utilizado para referirse a distintas cuestiones en relación a música. En un principio, para designar a sellos discográficos no comerciales, por ejemplo, Atlantic Records, que en origen publicaron grabaciones de artistas minoritarios de jazz, rhythm and blues y rock en torno al año $1950^{3}$. No obstante, su uso evolucionó y entre la década de 1970 y 1980 se empleó para referirse a la actitud de músicos vinculados con el punk que promulgaban su famoso "do it yourself" o hazlo tú mismo, a modo de reivindicación, en defensa de la música como expresión artística libre no condicionada por el número de ventas y las restricciones impuestas por las grandes multinacionales ${ }^{4}$.

Finalmente, durante la década de los ochenta comenzó a aplicarse para categorizar genéricamente a algunas bandas de noise/shoegaze norteamericanas, caso de Sonic Youth o Dinosaur Jr., y otras formaciones asociadas a las escena de Manchester ${ }^{5}$. En general estas agrupaciones asociadas al indie en los ochenta funcionaban en sellos pequeños, al margen de multinacionales, pero a diferencia de los casos anteriores, también poseían unas características musicales definidas, un sonido propio que comenzó a llamarse indie. Una de sus cualidades musicales más sobresalientes era la utilización de densos acordes de guitarra en lugar de riffs ${ }^{6}$. Esto quiere decir que en las composiciones primaban las armonías y el tratamiento de las texturas por encima de la creación melódica, a diferencia de lo que habitualmente se observa en aquellas producciones que persiguen como objetivo principal ocupar los primeros puestos de las listas de éxito y su difusión a través de radiofórmulas.

A partir de los noventa el público español sintetizó de alguna forma todas estas etapas de uso del término anglosajón y comenzó a emplear el denominador indie para referirse de manera indiscriminada a artistas independientes comercialmente que autoproducían su música; a pequeños sellos

\footnotetext{
${ }^{3}$ Ryan Moore: "Indie Rock", The Oxford Companion to Music. Oxford Music Online, Oxford University Press, http://www.oxfordmusiconline.com/subscriber/article/grove/music/A2241531?q=indie\&search=quick\&pos=4\&_start=1\#firsthit (consultado el 3-7-2017).

${ }^{4}$ R. Moore: "Indie Rock", The Oxford...

${ }^{5}$ Kenneth Gloag: "Indie", The Oxford Companion to Music. Oxford Music Online, Oxford University Press, http://www.oxfordmusiconline.com/subscriber/article/opr/t114/e3415 (consultado el 5-7-2017).

6 Allan F. Moore: "Indie Music", The Oxford Companion to Music. Oxford Music Online, Oxford University Press, en: http://www.oxfordmusiconline.com/subscriber/article/grove/music/46251?q=indie\& search=quick\&pos=5\&_start=1\#firsthit (consultado el 3-7-2017).
} 
discográficos que no estaban vinculados a multinacionales y a bandas locales de noise/shoegaze con un sonido similar al de las formaciones anglosajonas de los ochenta. Consecuentemente, en ese momento serían indies artistas como Sr. Chinarro, que producían y distribuían su música sin discográfica, en solitario o a través de colectivos de artistas como Karma; sellos nacionales con pequeño capital, como Elefant o Subterfuge, que trabajaban al margen de las grandes compañías y cuyas músicas eran minoritarias, al menos sobre el papel; y bandas como Penélope Trip, que mostraban ese sonido indie anglosajón desarrollado durante la década de los ochenta.

La prensa jugaría un papel crucial en este proceso de diversificación. Desde que el término aparece en la península y lo populariza el periodista Julio Ruiz, multitud de revistas como Rockdelux o Mondosonoro se dedican casi en exclusiva a este tipo de música y todo lo que la rodea.También en la radio hay un importante espacio reservado al indie, especialmente en Radio Nacional. Llama la atención que incluso aparecen programas como Viaje a los sueños polares en emisoras como Los 40 Principales, dedicadas a la difusión de las listas de artistas más vendidos ${ }^{7}$.

Poco a poco, se va configurando el mapa indie de España en el que sobresalen diferentes ciudades, caso de Gijón, Madrid o Granada y comunidades como el País Vasco. Localizaciones en las que no solo aparecen músicos indies. También encontramos un gran número de festivales dedicados a esta música, caso de Festimad, Festival Internacional de Benicassim (FIB) o Espárrago Rock, y locales y tiendas de discos dedicados a la difusión del indie. Un territorio formado durante la primera mitad de los noventa que demostraba que esta música se había asentado y extendido por todo el territorio español.

Una dispersión que conduce a una nueva tendencia desarrollada por algunos de los artistas nacionales vinculados con esta etiqueta de origen anglosajón. Durante la segunda mitad de los noventa y sobre todo a lo largo de la primera década del XXI, muchas de estas formaciones y músicos comienzan a introducir en las composiciones elementos distintivos de su región de procedencia buscando un indie propio, desarrollado fundamentalmente a través del folclore y las lenguas de cada territorio. Así encontramos a Lagartija Nick junto a Morente con Omega en 1996, Sr. Chinarro con Fuego Amigo (2005), Los Planetas y su Leyenda del espacio (2007) o Manel con Els millors professors europeus (2008).

Todo esto ha dado como resultado la creación de un indie español. Una categoría musical que, debido a su diversidad, cada vez engloba a un mayor número de seguidores pero que, a su vez, resulta tan dispar que en su

\footnotetext{
${ }^{7}$ Fernán del Val, Héctor Fouce: "De la apatía a la indignación. Narrativas del rock independiente español en época de crisis", Methaodos, Revista de ciencias sociales, 4, 1, 2016, p. 60.
} 
proceso constante de redefinición ha sido utilizado incluso en oposición a su idea original, para designar a bandas que editan sus discos en grandes multinacionales; bandas que tampoco poseen un sonido indie similar al de formaciones anglosajonas de los ochenta.

Llegados a este punto, el único denominador común entre todos los artistas asociados a la etiqueta indie en España se encuentra en la actitud. Una idea que recuerda a la propuesta punk de independencia, según la cual los músicos desarrollan una creación libre. Esta variable no solo es dificilmente cuantificable, sino que además queda en entredicho al leer testimonios de artistas como Juan Rodríguez Cervilla, "J”, líder de Los Planetas, quien afirmaba en una entrevista en relación a la multinacional con la que trabajaban: "hemos terminado contrato con Sony, así que ya no trabajamos por encargo [...] siempre nos han dejado libertad, aunque tienes la losa del contrato, de no puedo salirme de aquí hasta que acabe esto".

Esto corrobora que la hipótesis que Newman plantea sobre el cine independiente también es aplicable en música. Los artistas indies muestran dos caras diferentes. Por una parte buscan la autonomía, pero también el beneficio; la autenticidad pero sin perder de vista el empuje comercial; el arte sin el peso del comercio pero con el dinero suficiente para poder pagar la realización del arte, un enfrentamiento entre la necesidad y el deseo de la comunidad indie, estar alejado de todo lo que parece demasiado comercial ${ }^{9}$. Antonio Arias, líder de la banda de indie Lagartija Nick, lo subrayaba en una entrevista, afirmando "creo que tanto nosotros como otros muchos grupos tenemos un ojo puesto en el ambiente alternativo y otro puesto en la multinacional" 10 .

En resumen, el indie se ha convertido en una de las categorías más dificiles de definir dentro de las músicas populares urbanas de nuestro país, especialmente porque, en sentido estricto, el indie no debería considerarse un género o estilo musical ${ }^{11}$. La falta de trabajos académicos dedicados al análisis de esta etiqueta en España y la libertad con la que ha evolucionado el término durante el último cuarto de siglo, al ritmo que marcaban prensa divulgativa y público, ha potenciado este uso confuso.

Este artículo, por tanto, persigue dos objetivos. En primer lugar, definir el indie en España, delimitando su uso como género y como escena musical; en segundo lugar, comprender la dimensión de este fenómeno en expansión y resolver si nos encontramos ante un caso de oxímoron en música: el indie que triunfa en las listas de ventas.

\footnotetext{
${ }^{8}$ David Saavedra: "Los Planetas: entrevista del fin del mundo", Rockdelux, 2012, http://www.rockdelux.com/secciones/p/los-planetas-entrevista-del-fin-del-mundo.html (consultado el 10-7-2017).

${ }^{9}$ Michael Z. Newman: "Indie Culture: In Pursuit of the Authentic Autonomous Alternative", Cinema Journal, 48, 3, 2009, p. 26.

10 José Antonio Rueda: Independientes...
} 


\section{El indie en España, ¿género o escena?}

En una de las aproximaciones realizadas desde el ámbito académico a la música indie, Allan F. Moore afirma que como descriptor musical, el término indie no es demasiado útil. De hecho, desde la década de los ochenta, el sonido indie ha sido vinculado con otras muchas etiquetas como shoegaze o noise, por lo que incluso se podría prescindir de ella para hablar de música. No obstante, Moore define algunas características estilísticas que pueden ayudar a comprender el indie en su dimensión musical.

Según Moore, la música independiente mantiene muchas de las cualidades apreciables en otros subgéneros del rock, como el tipo de plantilla utilizado, la forma de las composiciones o las temáticas apreciables en sus letras. Sin embargo, presenta algunos rasgos diferenciadores. En primer lugar, su carácter introspectivo y la falta de proyección vocal de sus cantantes. A nivel de producción, la voz se mueve en el mismo plano que el resto de los instrumentos, incluso por debajo de estos. Su música se caracteriza por un cierto estatismo y se apoya sobre densos acordes de guitarra que pueden prolongarse a lo largo de la composición. Aparentemente, conseguir una melodía pegadiza no aparece entre sus prioridades ${ }^{12}$. En el indie prima la armonía y a nivel de producción el tratamiento de las texturas ${ }^{13}$. Además, las canciones indies habitualmente no poseen pasajes instrumentales virtuosísticos y en ellas, el peso melódico recae sobre la voz principal, aunque esto a veces puede llegar a ser inapreciable por el tratamiento que se da a las grabaciones. En estos casos la banda solo aporta soporte armónico y rítmico.

En este sentido, algunas formaciones españolas, caso de Los Planetas, pueden vincularse con esa música indie internacional definida por Moore. La formación granadina desde sus inicios ha utilizado dos o tres guitarras eléctricas, bajo eléctrico y batería. Además, puntualmente han sumado un teclado a la formación que aporta densidad a la textura y realiza, de manera eventual, pequeños motivos melódicos a los que se suma la voz, formando un muro de sonido compacto, similar al de bandas de shoegaze o indie rock como My Bloody Valentine, en el que es complicado discernir mediante una simple escucha los instrumentos que lo configuran ${ }^{14}$.

Este ejemplo no es único. Otros artistas como la banda Penélope Trip o Antonio Luque y su particular propuesta, Sr. Chinarro, han mostrado ideas similares.Valga como ejemplo la canción Quiromántico, aparecida en El porqué de mis peinados de 1997.

\footnotetext{
${ }^{11}$ F. Barrera: Hibridación, globalización..., p. 63.

12 Roy Shuker: Popular Music, the Key Concepts, Londres, Routledge, 1998, p. 171.

13 Allan F. Moore: "Indie Music", The Oxford..

${ }^{14}$ Fernando Barrera: Hibridación, globalización..., p. 129
} 
Sin embargo, dentro del diverso abanico de bandas asociadas al denominador indie en España, encontrar este tipo de recursos musicales no es habitual y hay pocos ejemplos de formaciones que cumplan con las escuetas indicaciones de Moore. De hecho, la mayoría de las formaciones vinculadas con el indie que más repercusión han tenido durante los últimos años se caracterizan precisamente por no tener unas características musicales comunes. Basta comparar cualquier canción de Manel, que hace indie en catalán, la banda sevillana Pony Bravo, indie con trazas de flamenco y la granadina Lori Meyers, indies que triunfan en una multinacional, para comprender que se trata de tres músicas totalmente diferentes. Incluso, como afirman Fouce y DelVal, los grupos de indie español cada vez piensan más en el público y han empezado a "cantar en castellano, a hacer estribillos claros y melodías bonitas. También empiezan a rebajar la distorsión"15.

Por todo ello, aunque pueda existir una música o sonido indie en España, al que se vincularían un reducido grupo de bandas, para comprender este término polisémico en toda su extensión debemos acudir al concepto de escena ${ }^{16}$.

Bennett y Peterson afirman que una escena local se forma en un lugar delimitado y un momento concreto y se define por las relaciones que se establecen entre sus participantes: productores, músicos y fans que comparten gustos musicales. Los miembros de una escena local absorben elementos culturales propios de otras escenas, reinterpretándolos y haciéndolos representativos de la escena local ${ }^{17}$. Asimismo se diferencian de los miembros de otras escenas no solo por su música. También juegan un papel importante cuestiones de consumo, como la forma de vestir. Se conforma así la identidad cultural de un determinado grupo social ${ }^{18}$.

En este sentido, la escena indie española tendría su germen antes incluso de que Julio Ruiz popularizara el término en Radio Nacional.Ya en la década de los ochenta se genera toda una escena independiente en sentido comercial en la que aparecen en nuestro país pequeños sellos discográficos, "fanzines, bares, grupos, revistas nuevas como Ruta 66 y fans metidos a discjockeys de radio" que escuchaban y difundían grupos extranjeros minoritarios

\footnotetext{
${ }^{15}$ Fernán del Val y Héctor Fouce: "De la apatía a...", p. 61.

${ }^{16} \mathrm{El}$ indie ha sido descrito como escena a nivel internacional por diversos autores como Holy Kruse en su trabajo "Local Identity and Independent Music Scenes, Online and Off", publicado en 2010 en el número 33 de la revista Popular Music and Society, pp. 625-639.

17 Richard A. Peterson, Andy Bennett: "Introducing Music Scenes", Music Scenes. Local, translocal and Virtual, Vanderbilt University press Bennett, 2004, p. 8.

${ }^{18}$ John O'Flynn: "National Identity and Music in Transition: Issues of Authenticity in Global Setting", Music, National Identity and the Politics of Location: Between the Global and the Local. Between the Global and the Local, Ian Biddle, Vanessa Knights (eds.), Hampshire, Ashgate, 2007, p. 31.
} 
dificiles de encontrar en el mercado español ${ }^{19}$. Incluso algunos de los artistas vinculados al indie en estos primeros años, en diversas entrevistas acudieron al tópico del músico transgresor, afirmando que el indie surgió a finales de los ochenta para romper con lo anterior, como oposición a La Movida, precisamente una escena ${ }^{20}$.

A lo largo de la década de los noventa, aparecerían un gran número de bandas y artistas que se sumarían a esta categoría, lo que a su vez provocaría un feedback, consiguiendo nuevos miembros y potenciando la creación de espacios y canales para el consumo indie, como festivales, emisoras de radio o tiendas especializadas.

Finalmente, a lo largo de los últimos años, la escena indie ha ido evolucionando, potenciando su cara más local, a través del uso de las distintas lenguas oficiales del país, que se han impuesto al inglés como idioma para la música indie española, como se aprecia en distintos artistas, caso de Emilio José, y la utilización de elementos propios de las músicas y estéticas populares de España, caso del flamenco, por ejemplo, utilizado por bandas y artistas como Pony Bravo o La Bien Querida.Así, podemos afirmar que existe una escena indie nacional formada a su vez por distintas escenas locales que ofrecen propuestas estéticas alternativas para abordar esta nacionalización de la música independiente.

\section{La música independiente y su dependencia}

Como afirma Hibbet, lo indie vende, es comercial porque desafia las estructuras sociales y económicas preexistentes, porque ser indie te distingue de la masa ${ }^{21}$. Este argumento ha sido apoyado por otros investigadores como David Hesmondhalgh, quien afirma que un movimiento artístico que desafía a las formas dominantes de la cultura acaba siendo absorbido por el sistema ${ }^{22}$.

Esto, que podría parecer una contradicción es en realidad un oxímoron, puesto que una premisa no invalida a la otra, sino que en su antagonismo, juntas originan un nuevo significado, una nueva vida para el indie.

En nuestro caso de estudio, no pasó mucho tiempo desde que naciera la escena nacional entre finales de los ochenta y principios de los noventa

\footnotetext{
${ }^{19}$ Nando Cruz: Pequeño circo. Historia oral del indie en España, Barcelona, Contraediciones, 2015, p. 40.

${ }^{20}$ Fernán del Val: "El rock español en los 80. Del underground a la institucionalización", Rock around Spain: Historia, industria, escenas y medios de comunicación, Kiko Mora y Eduardo Viñuela (eds.), Lleida, Edicions de la Universitat de Lleida, 2013, p. 46.

${ }^{21}$ Ryann Hibbet: "What is Indie Rock?", Popular Music and Society, 28, 1, 2005, p. 75.

22 David Hesmondhalgh, Leslie Meier: "Popular Music, Independence and the Concept of the Alternative in Contemporary Capitalism", Media Independence, Bennet, J. y Strange, N. (eds.), Londres, Routledge, 2015, p. 100
} 
y comenzara a fraguarse una industria vinculada a ella. Habitualmente se considera el concurso de maquetas de Discogrande del año 1992 como inicio oficial del indie en España. Esta independencia comercial duró poco, puesto que solo dos años después, en 1994, bandas como Los Planetas ya trabajaban para multinacionales y en esa primera mitad de la década de los noventa proliferaron multitud de eventos asociados a la escena, caso del FIB en 1995, promovido entre otros por Luis Calvo, creador de la discográfica indie Elefant. También otros sellos amateurs vinculados a estas músicas, caso de Green Ufos, por ejemplo, se profesionalizaron y empezaron a programar conciertos regularmente, a vivir de la música ${ }^{23}$.

En este momento, como afirma Ryan Moore, el indie funciona como un campo de cultivo en el que los cazatalentos encuentran nuevos artistas ${ }^{24}$. Incluso las multinacionales absorben compañías discográficas más pequeñas ${ }^{25}$. Así, esta escena marginal o alternativa se acerca a las corrientes comerciales, su sonido se estandariza y gana importancia en el ámbito de las músicas populares urbanas en España. Prueba de ello es la trayectoria de bandas como El Niño Gusano, que pasa de la independiente Grabaciones en el mar a RCA y BMG, durante la segunda mitad de los noventa; los asturianos Australian Blonde, de Subterfuge a BMG/RCA con Extra (1998); Chucho, que se movió entre Limbo Starr y Virgin; o Lori Meyers, que pasan del sello independiente Houston Party a la major Universal Music, entre los años 2004 y 2008.

La evolución de la escena no se detiene ahí. Este paso habitual de artistas de las compañías independientes a las compañías multinacionales entre finales de los noventa y principios del 2000 encontró su réplica en un gran aumento en el flujo de asistentes a los eventos de música indie. El FIB pasó de 15.000 asistentes en 1995 a 200.000 en $2009^{26}$. Asimismo, un vistazo a las listas de ventas de música de los últimos años permite comprobar que muchos de los artistas que se vinculan a la escena o etiquetan de manera habitual como indies se encuentran a la cabeza de los que más dinero generan para la música de nuestro país.Valgan como ejemplo los artistas internacionales The Black Keys, Radiohead o Imagine Dragons y nacionales como Silvia Pérez Cruz junto al músico y productor Raúl Fernández "Refree"27. De hecho, una de

\footnotetext{
${ }^{23}$ Juan Manuel Játiva: "Benicàssim, capital del pop alternativo", El País, 1995, https://elpais.com/diario/1995/08/04/cultura/807487201_850215.html (consultado el 14-7-2017).

${ }^{24}$ R. Moore: "Indie Rock", The Oxford...

${ }^{25}$ Asier Leoz: Rockdelux en la configuración de una escena musical indie en España (1990-2000), tesis doctoral, Bilbao, Universidad de Deusto, 2015, p. 366.

${ }^{26}$ EFE: "Agotadas las entradas para el FIB de los récords", El Mundo, 2009, http://www.elmundo.es/ elmundo/2009/06/05/valencia/1244210814.html (consultado el 14-7-2017).

${ }^{27}$ Pueden consultarse las listas de ventas españolas en la web de Promusicae, productores de música de España, http://www.promusicae.es/ (consultado el 14-7-2017).
} 
las bandas de indies más laureadas del territorio nacional, Los Planetas, alcanzó los primeros puestos de las listas de éxitos con Una ópera egipcia (2010) en su primera semana en el mercado, y su último disco, Zona temporalmente autónoma (2017), también se ha colado entre los más vendidos de nuestro país ${ }^{28}$.

Todo esto permite comprobar la existencia de una escena indie diversa, en la que se entremezclan valores dispares definidos por prensa, oyentes y músicos. Cuestiones musicales, como el sonido de la banda Los Planetas, formas de difusión comercial, caso de Pony Bravo y su sello discográfico y, en algunos casos, incluso bandas que no son independientes en relación a la industria discográfica ni poseen un sonido indie.

Con todo esto podemos afirmar que un cuarto de siglo después de su nacimiento en España, el grueso de bandas y artistas vinculados a la escena indie nacional no muestra patrones claros que ayuden a su categorización y definición como músicos indies. Además, tras el éxito obtenido durante la última década y su cada vez más evidente rol dentro de la industria discográfica, debemos preguntarnos ¿en qué medida el indie sigue siendo independiente?

\section{Conclusiones}

Las músicas populares se caracterizan por una enorme diversidad y una capacidad de transformación muy rápida. Esta potencialidad viene dada, especialmente en el caso de las músicas populares urbanas, por su estrecho vínculo con la sociedad, lo que provoca que todas las tendencias apreciables en la población, aunque sean minoritarias, presenten una réplica en los géneros urbanos. El indie en España es un claro ejemplo.

Desde su nacimiento, en las postrimerías de la década de los ochenta, el concepto música indie en España no ha parado de evolucionar y su estudio ha sido siempre problemático puesto que el término, usado en un contexto musical es polisémico y se ha empleado para referirse tanto a aspectos formales relacionados con la música de determinados artistas como a elementos sociales y económicos, sobre todo relacionados con la industria. De esta forma, hemos confundido habitualmente ambos aspectos, uniendo bajo un mismo denominador a bandas como Los Planetas, que poseen un sonido cercano al de formaciones indies internacionales, con otras agrupaciones como los sevillanos Pony Bravo, independientes en sentido comercial, puesto que distribuyen su música a través de su propio sello y al

${ }^{28}$ Resumen de las novedades aparecidas en las listas de éxito españolas, según Los40.com, en http://www.los40.com/actualidad/noticias/viva-la-vida-de-coldplay-es-el-disco-mas-vendido-de-lasemana/nota/1296548.aspx (consultado el 20-1-2014). 
margen de las grandes multinacionales. Incluso se ha etiquetado como indies a bandas que no tienen un sonido similar al de Los Planetas ni han sido nunca independientes en sentido comercial, trabajando para grandes multinacionales.

Esto ha dado como resultado la creación de un auténtico cajón desastre en el que tiene cabida prácticamente cualquier tipo de música. Además el término indie se aplica a otros muchos ámbitos de creación y consumo, como la literatura o el cine, lo que hace aún más complejo el estudio de esta categoría. No obstante, no se puede obviar que fuera del ámbito académico, es la propia sociedad la que decide incluso de manera contradictoria, lo que se asocia o no a una determinada etiqueta.

Por todo ello, la solución más plausible aunque no definitiva en el caso de la música indie en España pasa por la definición de escena. Comprender el indie no como género musical, sino como un entramado social que tiene lugar en nuestro país entre la década de los ochenta y la actualidad, en el que participan productores, músicos y seguidores que comparten gustos musicales y definen un conjunto de bandas y artistas que consideran representativos de sí mismos, lo que les distingue de la masa. El problema de esta idea radica en que no todos los miembros de la escena indie consideran indies a los mismos artistas, sino que se trata de un conjunto cambiante, lo que dificulta más si cabe su estudio.

Obviando estas cuestiones podemos afirmar que, aunque sigan existiendo artistas independientes que crean y difunden su música al margen de lo dictado por las listas de ventas, en general, la escena indie no es independiente. Por el contrario, se trata de una idea comercial realmente interesante y que durante el último cuarto de siglo no ha parado de crecer y generar beneficios para la industria musical, por definición, un oxímoron en música, el indie comercial. 